\title{
Review
}

\section{Pharmacological tools for lysophospholipid GPCRs: development of agonists and antagonists for LPA and S1P receptors}

\author{
Dong-Soon IM* \\ College of Pharmacy (BK21 Project) and Longevity Institute of Life Science and Technology, Pusan National University, Busan 609- \\ 735, Republic of Korea
}

Previous studies on lysophosphatidic acid (LPA) and sphingosine 1-phosphate (S1P) using various approaches have shown that both the molecules can act as intercellular signaling molecules. The discovery of the Edg subfamily of G-protein-coupled receptors (GPCRs) (later renamed $\mathrm{LPA}_{1-3}$ and $\mathrm{S}_{1} \mathrm{P}_{1-5}$ ) for these molecules has opened up a new avenue for pathophysiological research on lysophospholipids. Genetic and molecular studies on lysophospholipid GPCRs have elucidated pathophysiological impacts and roles in cellular signaling pathways. Recently, lysophospholipid GPCR genes have been used to develop receptor subtype-selective agonists and antagonists. The discovery of FTY720, a novel immune modulator, along with other chemical tools, has provided a means of elucidating the functions of each lysophospholipid GPCR on an organ and the whole body level. This communication attempts to retrospectively review the development of agonists and antagonists for lysophospholipid GPCRs, provide integrated information on pharmacological tools for lysophospholipid GPCR signaling, and speculate on future drug development.

Keywords: Iysophosphatidic acid; sphingosine 1-phosphate; agonist; antagonist; G-protein-coupled receptor; lysolipid

Acta Pharmacologica Sinica (2010) 31: 1213-1222; doi: 10.1038/aps.2010.135; published online 23 Aug 2010

\section{Discovery of GPCRs for LPA and S1P}

Lysophosphatidic acid (LPA) and sphingosine 1-phosphate (S1P) are two representative lysophospholipid mediators acting on G-protein-coupled receptors (GPCRs). LPA was first identified three decades ago as a factor affecting blood pressure, platelet aggregation, and smooth muscle contraction ${ }^{[1-5]}$ and rediscovered as a mitogenic serum lipid inducer of neurite retraction ${ }^{[6,7]}$. The name 'lyso' originates from lysis of blood cells; thus, the non-specific detergent action of LPA has been doubted. In 1996, the high potency (nmol/L range) and GPCR implications of LPA were finally connected to the discovery of the first LPA receptor (LPA 1 , formerly known as Edg-2 $)^{[8]}$. Subsequently, $\mathrm{LPA}_{2}$ and $\mathrm{LPA}_{3}$ were identified as members of the endothelial differentiation gene (Edg) subfamily of GPCRs $^{[9-11]}$. These three LPA receptors (Edg family) share a high homology with each other ${ }^{[12]}$.

Recently, the non-Edg family of LPA receptors, $\mathrm{LPA}_{4}$ (GPR23, p2y9), LPA $($ GPR92), and LPA 6 (p2y5), were reported as members of the purinergic GPCR cluster ${ }^{[13-19]}$. Using the

\footnotetext{
* To whom correspondence should be addressed.

E-mail imds@pusan.ac.kr

Received 2010-05-25 Accepted 2010-07-16
}

GPCR-Ga ${ }_{16}$ fusion expression system, Fujita's group reported GPR87 as another LPA receptor and $\mathrm{P}_{2} \mathrm{Y}_{10}$ as a dual receptor for both LPA and $S 1 P^{[20,21]}$. These results have not yet been confirmed by other research groups ${ }^{[22]}$. Instead, lysophosphatidylserine has been suggested to act as a ligand for $\mathrm{P}_{2} \mathrm{Y}_{10}$ without confirmation of LPA and S1P as ligands in NIH3T3 cells $^{[23]}$.

S1P was initially reported 15 years ago to be a second messenger, mediating an increase in calcium levels due to PDGF and IgE signaling ${ }^{[24,25]}$. The molecular target of S1P in the cytosol has not yet been identified. The initial finding of an S1P-induced calcium increase stimulated research in S1P biology and was linked to its recognition as an intercellular first messenger. The involvement of trimeric $\mathrm{G}$ proteins in S1Pinduced actions as well as pertussis toxin sensitivity strongly suggested the presence of S1P GPCRs in the plasma membrane ${ }^{[26-30]}$. The discovery of $\mathrm{S}_{1} \mathrm{P}_{1}$ (formerly known as Edg-1) in 1998, along with four other receptors $\left(\mathrm{S}_{\mathrm{P}} \mathrm{P}_{2-5}\right)$ of the $\mathrm{Edg}$ subfamily GPCRs, became a milestone in S1P biology ${ }^{[12,31-36]}$.

\section{Development of agonists and antagonists for LPA receptors}

Prior to receptor cloning, various aspects of LPA receptor 
structure-activity relationships had been studied: 1) fatty acid chain length and the presence of double bonds, 2) acyl and alkyl linkage, 3) stereo-selectivity on the $s n-2$ position, and 4) modification of phosphate groups ${ }^{[37]}$. Oleoyl LPA (18:1) was considered the optimum ratio to increase $\mathrm{Ca}^{2+}$ levels in A431 cells ${ }^{[38]}$. Alkyl LPA showed a better effect than acyl LPA in platelet aggregation assays and an equal effect in other responses ${ }^{[39,40]}$. Several groups reported a lack of stereo-selectivity on the sn-2 position of LPA. Early studies showed that modification of the phosphate group resulted in an absence of LPA responses ${ }^{[38,40]}$.

The initial synthetic approach to LPA response was characterized using platelet aggregation assays. Replacement of the glycerol backbone with amino acids resulted in the production of N-palmitoyl serine phosphatidic acid (NPSPA) and $\mathrm{N}$-palmitoyl tyrosine phosphatidic acid (NPTyrPA) ${ }^{[40]}$. These molecules were not active on platelet aggregation in humans, but did demonstrate activity as LPA receptor antagonists in Xenopus $^{[41]}$. Later, NPSPA and NPTyrPA were reported as partial agonists of mammalian LPA receptors ${ }^{[42,43]}$. Sugiura et al introduced the ethanolamine-based LPA mimetic, $N$-acyl aminoethanol phosphoric acid (NAEPA), as an equipotent agonist of platelet aggregation ${ }^{[40]}$. Later, research groups from the University of Virginia used NASPA and NAEPA as platforms to synthesize a series of VPC compounds ${ }^{[37,44]}$. The phosphonate analogue of NAEPA lost its platelet aggregation properties ${ }^{[40]}$, but methylene phosphonate LPA was equipotent to LPA as an inhibitor of forskolin-driven cAMP accumulation in rat $\mathrm{C} 6$ glioma cells ${ }^{[45]}$. Jalink et al synthesized various phosphonate analogues along with fatty alcohol phosphates and the methyl ester of LPA (lysophosphatidylmethanol, LPM), but could not show a significant impact of these compounds on $\mathrm{Ca}^{2+}$ increase in A431 cells ${ }^{[38]}$. Ironically, these chemicals turned out to be selective or non-selective agonists of cloned LPA receptors (see details below). In the early era of LPA biology, suramin and lysophosphatidylglycerol were used to demonstrate GPCR involvement in LPA responses ${ }^{[46]}$ and as an antagonist of LPA-induced $\mathrm{Ca}^{2+}$ responses in Jurkat $\mathrm{T}$ cells ${ }^{[47]}$, respectively.

\section{LPA GPCR agonists}

Since the discovery of the three-Edg family of LPA receptors, the development of selective receptor-subtype agonists and antagonists has accelerated. The optimal chain length and the presence of double bonds have been found to vary depending on receptor subtype. For example, $\mathrm{LPA}_{3}$ showed a preference for unsaturated LPA similar to oleoyl LPA ${ }^{[48]}$, whereas $\mathrm{LPA}_{6}$ showed a preference for 2-acyl LPA ${ }^{[19]}$. Synthesis of LPA derivatives with phosphonate or thiophosphate groups instead of the phosphate group showed receptor-subtype selective activity similar to 1-oleoyl-2-O-methyl-rac-glycerophosphothionate (OMPT), 1-O-acyl-a-fluoromethylenephosphonate, and a-hydroxymethylenephosphonate LPA analogues as $\mathrm{LPA}_{3}$ receptor selective agonists ${ }^{[49-52]}$. The phosphonate derivatives also provided a path toward the development of phosphataseresistant long-lasting LPA derivatives ${ }^{[53]}$. A dialkyl phospha- tidic acid (PA) 8:0 analog with a thiophosphate was reported as a potent and selective $\mathrm{LPA}_{3}$ agonist ${ }^{[54]}$, but later, agonistic activity on the $\mathrm{LPA}_{5}$ receptor and antagonistic activity on the $\mathrm{LPA}_{1,3}$ receptors was reported ${ }^{[5]}$. Based on computational modeling, dodecyl fatty alcohol phosphate was shown to be a specific $\mathrm{LPA}_{2}$ receptor agonist ${ }^{[56]}$, and later, oleoyl-thiophosphate was identified as a pan-agonist $\left(\mathrm{LPA}_{1-3}\right)^{[43]}$. A methylene phosphonate LPA analogue was reported as a selective $\mathrm{LPA}_{2}$ agonist, but it recently was shown to exert agonistic and antagonistic activities on $\mathrm{LPA}_{5}$ and $\mathrm{LPA}_{3}$, respectively ${ }^{[52,57]}$. T-15 (LPA 1 agonist) and T-13 (LPA 3 agonist) were both synthesized using a carbohydrate scaffold ${ }^{[58]}$. Darmstoff analogues were introduced as novel scaffolds for subtype-selective LPA receptor ligands ${ }^{[59]}$. Finally, octadecenyl phosphate was shown to be a selective agonist for $\mathrm{LPA}_{4}$ and $\mathrm{LPA}_{5}$ receptors ${ }^{[5]}$.

Although alkyl LPA has been shown to exert equipotent activity on each Edg-family LPA receptor compared to acyl $\mathrm{LPA}^{[60]}$, alkyl LPA was found to be more potent than acyl LPA in platelet aggregation responses ${ }^{[3]}$. Therefore, the Edgfamily LPA receptors were not able to account for the LPA response in platelet ${ }^{[6]]}$. Recently, non-Edg (purinergic) LPA receptors have been identified. The $\mathrm{LPA}_{5} / \mathrm{GPR} 92$ receptor has demonstrated alkyl preference and a presence in platelets ${ }^{[55]}$. Farnesyl diphosphate, $\mathrm{N}$-arachidonyl glycine, and carba-cyclic phosphatidic acid were shown to be selective agonists for $\mathrm{LPA}_{5}{ }^{[55,62,63]} . \mathrm{LPA}_{6} / \mathrm{p} 2 \mathrm{y} 5$ showed a preference for 2-acyl LPA over 1-acyl LPA ${ }^{[19]}$. Alkyl-OMPT, an $\mathrm{LPA}_{3}$ agonist, and 2-linoleoyl LPA showed better agonistic effects on the $\mathrm{LPA}_{6}$ receptor than 2-oleoyl LPA showed ${ }^{[19,64]}$. In contrast, the methyl ester of LPA (LPM) was shown to be a pan-agonist for $\mathrm{LPA}_{1-5}$ although it was less potent than $\mathrm{LPA}^{[65]}$.

\section{LPA GPCR antagonists}

The development of LPA derivatives with a bulky group on the $s n-2$ position has demonstrated the stereo-selectivity of LPA receptors and has led to the further development of the $\mathrm{LPA}_{1} / \mathrm{LPA}_{3}$ selective antagonists VPC12249 and VPC $32183^{[66,67]}$. 2-Pyridyl-containing phosphonate was developed as a non-hydrolyzable $\mathrm{LPA}_{3}$ receptor antagonist ${ }^{[68]}$. Dioctylglyceropyrophosphate (DGPP) and Ki16425 were determined to be selective $\mathrm{LPA}_{1} / \mathrm{LPA}_{3}$ antagonists following screening of available lipid and non-lipid molecules ${ }^{[69,70]}$. The thiophosphate version of PA 8:0 was shown to be the most potent $\mathrm{LPA}_{3}$ antagonist, whereas the dialkyl PA 8:0 analog with a thiophosphate was shown to be a potent and selective $\mathrm{LPA}_{3,5}$ agonist, as mentioned above ${ }^{[54,55]}$. Tetradecyl-phosphonate was identified as a pan-antagonist $\left(\mathrm{LPA}_{1-3}\right)^{[43]}$. Furthermore, farnesyl phosphate and farnesyl diphosphate potently and selectively blocked the $\mathrm{LPA}_{3}$-mediated $\mathrm{Ca}^{2+}$ increase ${ }^{[71]}$, although farnesyl diphosphate is a $\mathrm{LPA}_{5}$ selective agonist, as mentioned above ${ }^{[55]}$. T-14, which used a carbohydrate scaffold, was shown to be an $\mathrm{LPA}_{3}$ antagonist ${ }^{[58]}$.

Recently, virtual screening identified non-lipid $\mathrm{LPA}_{3}$ antagonist NSC161613, $\mathrm{LPA}_{2}$ antagonist H2L5186303, $\mathrm{LPA}_{4,5}$ antagonist 5987411, and $\mathrm{LPA}_{1,6}$ antagonist $5765834^{[55,72]}$. An LPA analogue of a-bromomethylene phosphonate was reported to act 
as a pan-antagonist to $\mathrm{LPA}_{1-4}$ and an autotoxin inhibitor ${ }^{[52,73]}$.

\section{Pharmacological tools for LPA GPCR signaling}

Commercially available chemicals for studying LPA receptor subtypes are currently in development, although the effects of previously developed chemicals on recently identified nonEdg LPA receptors have not been completely verified (Figure 1). For $\mathrm{LPA}_{1}$ or $\mathrm{LPA}_{3}$ receptor signaling, a combined application of $\mathrm{LPA}_{1,3}$ antagonists such as VPC12249, VPC32193, DGPP, and Ki16425 and $\mathrm{LPA}_{3}$ agonists such as OMPT and a-fluoromethylene phosphonate would be more favorable. For $\mathrm{LPA}_{2}$ receptor signaling, dodecyl phosphate is an adequate $\mathrm{LPA}_{2}$ selective agonist. For $\mathrm{LPA}_{5}$ receptor, farnesyl diphosphate could be used as a selective agonist. For $\mathrm{LPA}_{6}$ receptor, alkyl OMPT, an $\mathrm{LPA}_{3,6}$ agonist, could be used in combination with $\mathrm{LPA}_{1,3}$ antagonists (Table 1).

\section{Development of agonist and antagonist for S1P receptors} In contrast to LPA, the structure-activity relationship of S1P has a very short history. Using cloned S1P receptors, sphinganine-1-phosphate (dihydro-S1P) and sphingosylphosphorylcholine (SPC) were shown to be equipotent and far less potent on each S1P receptor ${ }^{[36,74-76]}$.

\section{S1P GPCR agonists}

Following the cloning of the S1P receptors, the development of S1P agonists and antagonists began. The importance of the D-erythro configuration of S1P was demonstrated using the cloned receptors ${ }^{[7]}$. The linkage of the immune modulator FTY720 to S1P receptors, however, boosted this area of research and opened a new direction for S1P biology ${ }^{[78-80]}$. Lymphopenia induction by inhibiting lymphocyte egress from lymphoid organs was shown to be mediated through the $\mathrm{S}_{1} \mathrm{P}_{1}$ receptor $^{[81]}$. High-throughput screening (HTS) of an available chemical library showed that SEW2871 acted as an in vivo active heterocyclic $S 1 P_{1}$ selective agonist ${ }^{[81,82]}$ and compound 26 was synthesized as a potent 3,5-diphenyl-12,4-oxadiazole $\mathrm{S} \mathrm{P}_{1}$ agonist ${ }^{[83]}$. Later, using ultra-HTS, 3,5-diaryloxadiaxole (CYM5181) and dicyclohexylamide were found to be selective agonists for $\mathrm{S}_{1} \mathrm{P}_{1}$ and $\mathrm{S}_{1} \mathrm{P}_{3}$, respectively ${ }^{[84]}$. Using computational modeling, CYM-5442 was developed as an $\mathrm{S} \mathrm{P}_{1}$ selective agonist that was more potent than CYM5181 ${ }^{[85]}$. AUY954, an aminocarboxylate analogue of FTY720, was also introduced as an $\mathrm{S}_{1} \mathrm{P}_{1}$ selective agonist ${ }^{[86]}$. VPC01091, a cyclized analogue of FTY720, was shown to act as an orally active $\mathrm{S1P}_{1}$ agonist and an $\mathrm{S} \mathrm{P}_{3}$ antagonist ${ }^{[87]}$. KRP-203 is a pro-drug immune modulator similar to FTY720; the phosphorylated form of KRP-203 was shown to be a selective $\mathrm{S} \mathrm{P}_{1}$ agonist ${ }^{[88,89]}$. Constrained azacyclic analogues of FTY720 showed selective agonist activities on $\mathrm{S} \mathrm{P}_{4}$ and $\mathrm{S}_{1} \mathrm{P}_{5}$ receptors ${ }^{[90]}$. Finally, phytosphingosine1-phosphate was shown to act as a potent and selective agonist on the $\mathrm{S}_{1} \mathrm{P}_{4}$ receptor ${ }^{[76]}$.

\section{S1P GPCR antagonists}

Suramin was temporarily used as an $\mathrm{S} \mathrm{P}_{3}$ antagonist ${ }^{[75,91]}$. Human $\mathrm{S1P}_{5}$ was also reported to be sensitive to suramin and its analogue $\mathrm{NF} 023^{[92]}$.

Following screening of an available chemical library, JTE013, a pyrazopyridine derivative, was identified as an $\mathrm{S1P}_{2}$ antagonist ${ }^{[33,94]}$. Modification of the FTY720-phosphate structure led to the development of VPC23019 and VPC25239 as selective $\mathrm{S} \mathrm{P}_{1} / \mathrm{S}_{1} \mathrm{P}_{3}$ antagonists ${ }^{[95]}$. As mentioned above, VPC01091 is an orally active $\mathrm{S1P}_{1}$ agonist and $\mathrm{S}_{1} \mathrm{P}_{3}$ antagonist ${ }^{[87]}$. W146, hexyl phenyl amide phosphonate, was found to
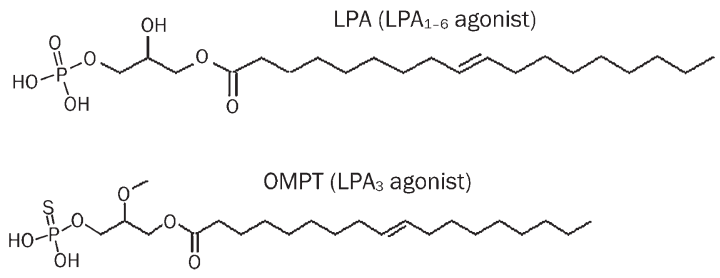
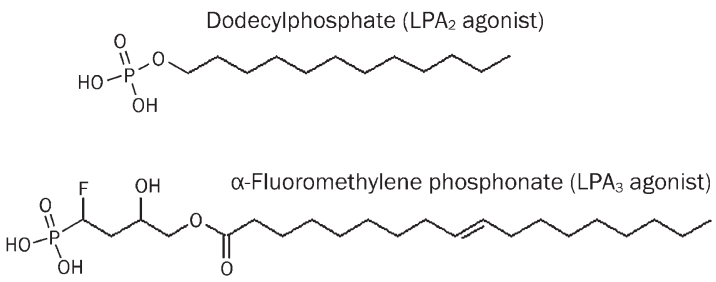
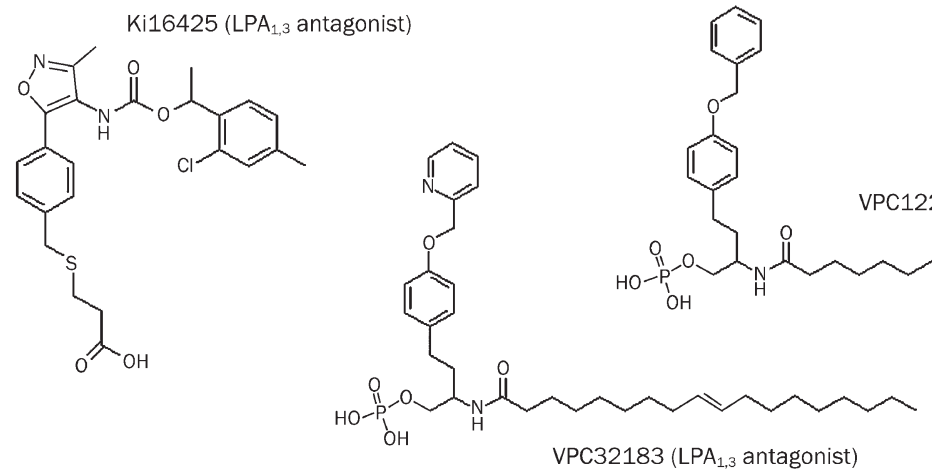

PC12249 ( $\mathrm{LPA}_{1,3}$ antagonist)

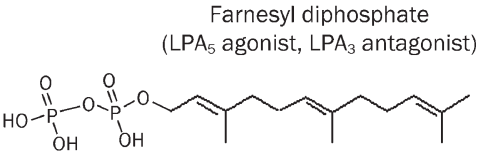

Echelon bioscience, Enzo Life sciences, and Sigma-Aldrich. 
Table 1. Agonistic and antagonistic characters of each compound on each LPA receptor. Numbers ( $n m m o l / L)$ mean $E_{50}$ or $K_{D}$ values for agonists and $\mathrm{IC}_{50}$ or $\mathrm{K}_{1}$ values for antagonists.

\begin{tabular}{|c|c|c|c|c|c|c|}
\hline Name & $\mathrm{LPA}_{1}$ & $\mathrm{LPA}_{2}$ & $\mathrm{LPA}_{3}$ & $\mathrm{LPA}_{4}$ & $\mathrm{LPA}_{5}$ & $\mathrm{LPA}_{6}$ \\
\hline Ki16425 ${ }^{[70]}$ & $\begin{array}{l}\text { Antagonist } \\
\text { (250 nmol/L) }\end{array}$ & $\begin{array}{l}\text { Antagonist } \\
\text { (5600 nmol/L) }\end{array}$ & $\begin{array}{l}\text { Antagonist } \\
(360 \mathrm{nmol} / \mathrm{L})\end{array}$ & & & \\
\hline DGPP [69] & $\begin{array}{l}\text { Antagonist } \\
\text { (6600 nmol/L) }\end{array}$ & & $\begin{array}{l}\text { Antagonis } \\
(106 \mathrm{nmol} / \mathrm{L})\end{array}$ & & & \\
\hline VPC32183 $3^{[67]}$ & $\begin{array}{l}\text { Antagonist } \\
\text { (109 nmol/L) }\end{array}$ & & $\begin{array}{l}\text { Antagonist } \\
(175 \mathrm{nmol} / \mathrm{L})\end{array}$ & & & \\
\hline 2-Pyridyl phosphonate ${ }^{[68]}$ & & & Antagonist & & & \\
\hline Thiophosphatidic acid 8:0 $0^{[54]}$ & $\begin{array}{l}\text { Antagonist } \\
(360 \mathrm{nmol} / \mathrm{L})\end{array}$ & & $\begin{array}{l}\text { Antagonist } \\
(5 \mathrm{nmol} / \mathrm{L})\end{array}$ & & & \\
\hline H2L5186303 $3^{[72]}$ & & $\begin{array}{l}\text { Antagonist } \\
(7.2 \mathrm{nmol} / \mathrm{L})\end{array}$ & $\begin{array}{l}\text { Antagonist } \\
(310 \mathrm{nmol} / \mathrm{L})\end{array}$ & & & \\
\hline $5987411^{[55]}$ & & & & $\begin{array}{l}\text { Antagonist } \\
\text { (741 nmol/L) }\end{array}$ & $\begin{array}{l}\text { Antagonist } \\
\text { (1300 nmol/L) }\end{array}$ & \\
\hline $5765834^{[55]}$ & $\begin{array}{l}\text { Antagonist } \\
(48 \mathrm{nmol} / \mathrm{L})\end{array}$ & & & & $\begin{array}{l}\text { Antagonist } \\
\text { (292 nmol/L) }\end{array}$ & \\
\hline $\begin{array}{l}\alpha \text {-bromomethylene } \\
\text { phosphonate }^{[52,55,73,117]}\end{array}$ & $\begin{array}{l}\text { Antagonist } \\
(751 \mathrm{nmol} / \mathrm{L})\end{array}$ & $\begin{array}{l}\text { Antagonist } \\
\text { (304 nmol/L) }\end{array}$ & $\begin{array}{l}\text { Antagonist } \\
(380 \mathrm{nmol} / \mathrm{L})\end{array}$ & $\begin{array}{l}\text { Antagonist } \\
(167 \mathrm{nmol} / \mathrm{L})\end{array}$ & Weak agonist & \\
\hline Tetradecyl-phosphonate ${ }^{[43]}$ & $\begin{array}{l}\text { Antagonist } \\
\text { (10000 nmol/L) }\end{array}$ & $\begin{array}{l}\text { Antagonist } \\
\text { (5500 nmol/L) }\end{array}$ & $\begin{array}{l}\text { Antagonist } \\
\text { (3100 nmol/L) }\end{array}$ & & & \\
\hline $\begin{array}{l}\alpha \text {-fluoromethylene } \\
\text { phosphonate }^{[51]} \\
\alpha \text {-hydroxymethylene- } \\
\text { phosphonate }^{[52]}\end{array}$ & Weak agonist & Weak agonist & $\begin{array}{l}\text { Agonist } \\
(0.5 \mathrm{nmol} / \mathrm{L}) \\
\text { Agonist } \\
\text { (393 nmol/L) }\end{array}$ & & & \\
\hline Compound 8bo ${ }^{[121]}$ & $\begin{array}{l}\text { Agonist } \\
(9.1 \mathrm{nmol} / \mathrm{L})\end{array}$ & & $\begin{array}{l}\text { Agonist } \\
(123 \mathrm{nmol} / \mathrm{L})\end{array}$ & & & \\
\hline $\begin{array}{l}\text { Dialkyl thiophosphatidic } \\
\text { acid } 8: 0^{[54,55]}\end{array}$ & $\begin{array}{l}\text { Agonist } \\
\text { (695 nmol/L) } \\
\text { Antagonist } \\
\text { (382 nmol/L) }\end{array}$ & $\begin{array}{l}\text { Agonist } \\
\text { (5720 nmol/L) }\end{array}$ & $\begin{array}{l}\text { Agonist } \\
\text { (3 nmol/L) } \\
\text { Antagonist } \\
\text { (184 nmol/L) }\end{array}$ & & Agonist & \\
\hline Dodecylphosphate ${ }^{[56]}$ & & $\begin{array}{l}\text { Agonist } \\
\text { (700 nmol/L) }\end{array}$ & $\begin{array}{l}\text { Antagonist } \\
(90 \mathrm{nmol} / \mathrm{L})\end{array}$ & & & \\
\hline $\begin{array}{l}\alpha \text {-methylene } \\
\text { phosphonate }^{[52,55]}\end{array}$ & & $\begin{array}{l}\text { Agonist } \\
\text { (>281 nmol/L) } \\
\text { Antagonist } \\
(1420 \mathrm{nmol} / \mathrm{L})\end{array}$ & & $\begin{array}{l}\text { Weak agonist } \\
(3900 \mathrm{nmol} / \mathrm{L})\end{array}$ & Agonist & \\
\hline $\mathrm{T} 15^{[58]}$ & $\begin{array}{l}\text { Agonist } \\
\text { (5 nmol/L) }\end{array}$ & & $\begin{array}{l}\text { Agonist } \\
(50 \mathrm{nmol} / \mathrm{L})\end{array}$ & & & \\
\hline
\end{tabular}




\begin{tabular}{|c|c|c|c|c|c|c|}
\hline Name & $\mathrm{LPA}_{1}$ & $\mathrm{LPA}_{2}$ & $\mathrm{LPA}_{3}$ & $\mathrm{LPA}_{4}$ & $\mathrm{LPA}_{5}$ & $\mathrm{LPA}_{6}$ \\
\hline $\mathrm{T} 13^{[58]}$ & $\begin{array}{l}\text { Week agonist } \\
\text { (500 nmol/L) }\end{array}$ & & $\begin{array}{l}\text { Agonist } \\
(0.5 \mathrm{nmol} / \mathrm{L})\end{array}$ & & & \\
\hline Octadecenyl phosphate ${ }^{[55]}$ & & & & $\begin{array}{l}\text { Agonist } \\
\text { (608 nmol/L) }\end{array}$ & Agonist & \\
\hline $\operatorname{NPSPA}^{[11,43,122]}$ & $\begin{array}{l}\text { Weak agonist } \\
\text { (1850 nmol/L) }\end{array}$ & Weak agonist & $\begin{array}{l}\text { Weak agonist } \\
(1600 \mathrm{nmol} / \mathrm{L})\end{array}$ & & & \\
\hline$N^{N A E P A}{ }^{[11]}$ & Agonist & Agonist & Weak agonist & & & \\
\hline Oleoyl-thiophosphate ${ }^{[43]}$ & $\begin{array}{l}\text { Agonist } \\
\text { (193 nmol/L) }\end{array}$ & $\begin{array}{l}\text { Agonist } \\
\text { (244 nmol/L) }\end{array}$ & $\begin{array}{l}\text { Agonist } \\
\text { (546 nmol/L) }\end{array}$ & & & \\
\hline LPM $^{[65]}$ & Agonist & Agonist & Agonist & Agonist & Agonist & \\
\hline
\end{tabular}

be a selective $\mathrm{S1P}_{1}$ antagonist ${ }^{[96]}$. VPC44116, an octyl analogue of W146 and $y$-aminophosphonate analogue of VPC23019, antagonized lymphopenia and lung permeability via the $\mathrm{S}_{1} \mathrm{P}_{1}$ receptor ${ }^{[97]}$. SB64146 was reported to act as an inverse agonist on the $\mathrm{S}_{1} \mathrm{P}_{1}$ receptor ${ }^{[98]}$. Ascotricins $\mathrm{A}$ and $\mathrm{B}$ were isolated from a cultured broth of a fungus identified as Ascotricha chartarum and shown to inhibit the $\mathrm{S}_{1} \mathrm{P}_{1}$ receptor and S1Pmediated HUVEC migration ${ }^{[99]}$. Sankyo Co synthesized compound lead 2 (CL2), 2-(4-ethoxyphenoxy)-5-(3-octadecyl-5oxo-4,5-dihydro-1H-pyrazol-1-yl) benzenesulfonate, which antagonized the $\mathrm{S}_{1} \mathrm{P}_{1}>\mathrm{S}_{1} \mathrm{P}_{3}>\mathrm{S}_{1} \mathrm{P}_{2}$ receptors ${ }^{[100]}$. Human $\mathrm{S}_{1} \mathrm{P}_{1}$ receptor-selective antagonist and agonist effects of a rat monoclonal antibody (4B5.2) in vivo were also reported ${ }^{[101]}$. Using a 3D database search, BML-241, 2-alkylthiazolidine-4-carboxylic acid, was found to act as an $\mathrm{S1P}_{3}$ antagonist, but its selectivity and potency were not recapitulated in CHO-K1 cells expressing the $\mathrm{S}_{1} \mathrm{P}_{3}$ receptor ${ }^{[102,103]}$. A pharmacophore-based design of an $\mathrm{S1P}_{3}$ antagonist with a 3,4-dialkyoxybenzophenone scaffold was suggested ${ }^{[104]}$.

\section{Pharmacological tools for S1P GPCR signaling}

Commercially available tools for studying S1P receptor sub- types are highlighted in Figure 2. For $\mathrm{S}_{1} \mathrm{P}_{1}$ receptor signaling, CYM-5442 or SEW2871, both potent selective $\mathrm{S}_{1} \mathrm{P}_{1}$ agonists, and $\mathrm{W} 146$, a selective $\mathrm{S}_{1} \mathrm{P}_{1}$ antagonist, should be sufficient to elucidate $\mathrm{S}_{1} \mathrm{P}_{1}$ receptor involvement. $\mathrm{S}_{1} \mathrm{P}_{2}$ receptor signaling could be dissected using JTE-013, an $\mathrm{S}_{1} \mathrm{P}_{2}$ selective antagonist. For $\mathrm{S}_{1} \mathrm{P}_{3} \mathrm{GPCR}$ signaling, a combined application of an $\mathrm{S1P}_{1,3}$ antagonist (VPC23019) and $\mathrm{S1P}_{1}$ antagonist (W146) or $\mathrm{S}_{1} \mathrm{P}_{1}$ agonist (CYM-5442) could be useful. Phytosphingosine 1-phosphate, an $\mathrm{S} \mathrm{P}_{4}$ selective agonist, could be used to study

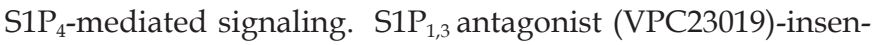
sitive, $\mathrm{S}^{\mathrm{P}_{2}}$ antagonist (JTE-013)-insensitive, $\mathrm{S} \mathrm{P}_{4}$ agonist-nonresponsive, and S1P- or FTY720-phosphate-sensitive signaling might be interpreted as $\mathrm{S} \mathrm{P}_{5}$ receptor or unidentified S1P receptor signaling (Table 2).

\section{Development of drugs acting on lysophospholipid GPCRs}

Prior to the molecular cloning of GPCRs for LPA and S1P, a number of studies were conducted with the primary goal of determining the functions of lipid mediators at both the cellular and the organ level. These functions included platelet aggregation, smooth muscle contraction, and cell proliferation, among others ${ }^{[1-6,105]}$. The discovery of GPCRs allowed signal<smiles>CCCCCCCCCCCC/C=C/C(O)C(N)COP(=O)(O)O</smiles><smiles>CCOc1ccc(-c2nc(-c3ccc4c(c3)CCC4NCCO)no2)cc1OCC</smiles><smiles>CCCCCCCCCCCCC(O)C(O)C(O)C(N)COP(=O)(O)O</smiles>

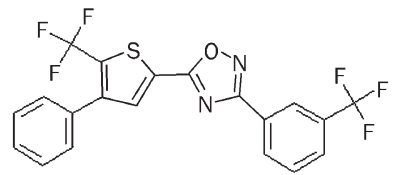

SEW2871 (S1P 1 agonist)

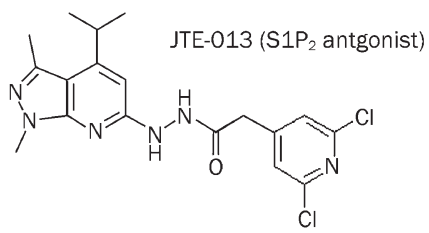

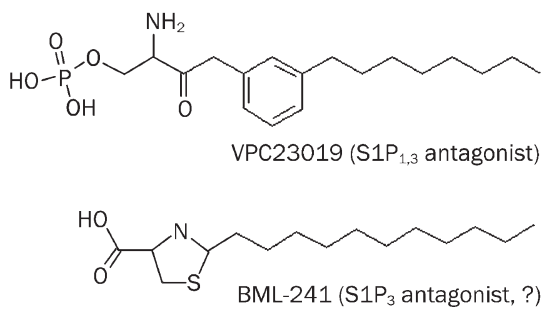

Figure 2. Structures of commercially available agonists and antagonists for S1P GPCR signaling. Sources are Avanti polar lipid, Biomol international, Cayman chemical, Echelon bioscience, Enzo Life sciences, Sigma-Aldrich, and Tocris. 
Table 2. Agonistic and antagonistic characters of each compound on each S1P receptor. Numbers (nmol/L) mean $E_{50}$ or $K_{D}$ values for agonists and $\mathrm{IC}_{50}$ or $\mathrm{K}_{1}$ values for antagonists.

\begin{tabular}{|c|c|c|c|c|c|}
\hline Name & $\mathrm{SIP}_{1}$ & $\mathrm{SIP}_{2}$ & $\mathrm{SIP}_{3}$ & $\mathrm{SIP}_{4}$ & $\mathrm{SIP}_{5}$ \\
\hline AUY954 $4^{[86]}$ & Agonist (1.2 nmol/L) & & Agonist (1210 nmol/L) & & Agonist (340 nmol/L) \\
\hline CYM-5442 $2^{[85]}$ & Agonist (1.2 nmol/L) & & & & \\
\hline CYM-5181 $1^{[84]}$ & Agonist (3.4 nmol/L) & & & & \\
\hline SEW2871 ${ }^{[81]}$ & Agonist (13 nmol/L) & & & & \\
\hline Compound $26^{[83]}$ & Agonist $(0.6 \mathrm{nmol} / \mathrm{L})$ & & Agonist (12000 nmol/L) & Agonist (70 nmol/L) & Agonist (1.0 nmol/L) \\
\hline Compound $12^{[90]}$ & & & & Agonist (7.4 nmol/L) & Agonist (10.2 nmol/L) \\
\hline Compound $18^{[90]}$ & & & & Agonist (16.8 nmol/L) & Agonist (5.8 nmol/L) \\
\hline VPC44116 $6^{[97]}$ & Antagonist (30 nmol/L) & & Antagonist (300 nmol/L) & & $\begin{array}{l}\text { Partial agonist } \\
\text { (33 nmol/L) }\end{array}$ \\
\hline W146 $6^{[96,106]}$ & Antagonist (36 nmol/L) & & & & \\
\hline SB649146 $6^{[98]}$ & Antagonist (300 nmol/L) & & & & \\
\hline VPC23019 $9^{[95]}$ & Antagonist (13.8 nmol/L) & & Antagonist (1175 nmol/L) & Agonist (263 nmol/L) & $\begin{array}{l}\text { Partial agonist } \\
(85.1 \mathrm{nmol} / \mathrm{L})\end{array}$ \\
\hline VPC25239 $9^{[95]}$ & Antagonist (13.4 nmol/L) & & Antagonist (97.7 nmol/L) & Agonist (166 nmol/L) & $\begin{array}{l}\text { Partial agonist } \\
\text { (11.5 nmol/L) }\end{array}$ \\
\hline $\operatorname{CL} 2^{[100]}$ & Antagonist (4400 nmol/L) & Antagonist (37000 nmol/L) & Antagonist (6700 nmol/L) & & \\
\hline VPC01091-P ${ }^{[87]}$ & Agonist $(6.6 \mathrm{nmol} / \mathrm{L})$ & & Antagonist & Agonist & Partial agonist \\
\hline Ascotricins & Antagonist $(8200 \mathrm{nmol} / \mathrm{L}$, & & & & \\
\hline$A$ and $B^{[99]}$ & $1800 \mathrm{nmol} / \mathrm{L})$ & & & & \\
\hline JTE-013 $3^{[93]}$ & & Antagonist (17 nmol/L) & & & \\
\hline $\mathrm{BML}-241^{[102,103]}$ & & & Antagonist (?) & & \\
\hline TY-52156 $6^{[123]}$ & & & Antagonist (110 nmol/L) & & \\
\hline DS-SG-44 ${ }^{[124]}$ & Agonist & Agonist & Agonist & & \\
\hline KRP-203-P $[88,89]$ & Agonist $(0.8 \mathrm{nmol} / \mathrm{L})$ & & & Agonist (9.6 nmol/L) & \\
\hline PhytoS1P $P^{[76]}$ & & & & Agonist (1.6 nmol/L) & \\
\hline DihydroS1 $\mathrm{P}^{[36,75,76]}$ & Agonist & Agonist & Agonist & Agonist $(8.6 \mathrm{nmol} / \mathrm{L})$ & Agonist \\
\hline $\mathrm{SPC}^{[34,36,75]}$ & Partial agonist & Partial agonist & Partial agonist & Partial agonist & Partial agonist \\
\hline FTY720-P ${ }^{[78,79]}$ & $\begin{array}{l}\text { Agonist }(6.3 \mathrm{nmol} / \mathrm{L}) \text {, } \\
\text { functional antagonist }\end{array}$ & & Agonist (4.0 nmol/L) & Agonist (6.3 nmol/L) & Agonist ( $6.3 \mathrm{nmol} / \mathrm{L})$ \\
\hline AFD-R ${ }^{[79]}$ & Agonist (2.5 nmol/L) & & Agonist (4.0 nmol/L) & Agonist (4.0 nmol/L) & Agonist (1.3 nmol/L) \\
\hline
\end{tabular}

transduction studies to proceed in cells over-expressing the receptors and in receptor knock-down transgenic mouse models $^{[106-109]}$. The discovery of the pathophysiological significance of LPA and S1P, particularly on each receptor subtype, would contribute favorably to new drug development. In developing new medications acting on LPA or S1P receptors, subtype selectivity would be a major issue along with potency and efficacy to avoid side effects and ensure drug safety.

FTY720 was initially developed as an immune modulator for organ transplant patients ${ }^{[110]}$. At present, this compound is under clinical study for the treatment of multiple sclerosis ${ }^{[111]}$. Amira Pharmaceuticals reported $\mathrm{LPA}_{1}$ selective antagonist (4' -\{4-[(R)-1-(2-chloro-phenyl)-ethoxycarbonylamino]-3methyl-isoxazol-5-yl\}-biphnyl-4-yl)-acetic acid, AP2966, which showed good therapeutic potential in idiopathic pulmonary fibrosis and good pharmacokinetic profiles, including oral bioavailability ${ }^{[112]}$. Pfizer global research and development introduced the $\mathrm{S}_{1} \mathrm{P}_{1}$ selective agonists PF-A and PF-B. These agonists resulted in lymphopenia in rats and monkeys similar to FTY720 and reduced collagen-induced arthritis ${ }^{[13]}$. Using a different approach, Lpath Inc. recently introduced monoclonal antibodies against S1P and LPA. Humanized anti-S1P monoclonal antibody $(\mathrm{mAb})$ sonepcizumab blocked the tumorigenic effect of S1P produced by cancer cells as well as the angiogenic effect induced during pathological angiogenesis ${ }^{[114,115]}$. The LPA receptor pan-antagonist $\left(\mathrm{LPA}_{1-4}\right)$ and autotoxin inhibitor, a-bromomethylene phosphonate LPA analogue, was shown to be an excellent anti-cancer agent $^{[116,117]}$.

\section{Closing remarks}

Following the initial discoveries of LPA activity 30 years ago and S1P activity 15 years ago, a dark dawn era in lysophospholipid biology occurred due to the lack of identifiable targets. The discovery of the Edg-family GPCRs for LPA and S1P shined light on this field. The first meeting that focused on lysophospholipid biology was a New York Academy of Science meeting conducted in 1999 at Rockefeller University ${ }^{[118]}$. Since 2001, FASEB summer research conferences on lysophospholipids have been held biannually. Lysophospholipid receptor nomenclature has been systematically assigned ${ }^{[19]}$, and more LPA receptors (purinergic or non-Edg LPA recep- 
tors) are being discovered each year. Every two years, we have had exciting findings including the linkage of FTY720 to the S1P receptor, discovery of an autotoxin as a LPA-producing lysoPLD, lysolipid-sensitive proton-sensing GPCRs (OGR1 subfamily), and finally, the development of new chemicals. This review integrates accumulated information regarding pharmacological tools for lysophospholipid GPCR signaling to compare their characteristics and provides valuable information such as available chemical sources. Using a combination of receptor expression patterns in each organ or cell, these pharmacological tools might prove useful in defining the pathophysiological impact and significance of lysophospholipids. As mentioned above, the control of lysophopholipid functions using specific agonists or antagonists will contribute toward novel drug development. At a 2009 FASEB meeting in Carefree (Arizona, USA), we began to see several of the chemicals described here, in addition to FTY720, being used in clinical applications ${ }^{[112-114,116]}$. We now know more about many things than ever before. It is very likely that in the near future, the agonists/antagonists for LPA or S1P receptors will be on the market commercially and that there will be a section on lysophospholipid GPCRs in every basic pharmacology textbook.

\section{Acknowledgements}

This work was supported by the National Research Foundation, 2010 Korea-Japan Joint Research Grant (2010-616E00015).

\section{References}

1 Gerrard JM, Kindom SE, Peterson DA, Peller J, Krantz KE, White JG. Lysophosphatidic acids. Influence on platelet aggregation and intracellular calcium flux. Am J Pathol 1979; 96: 423-38.

2 Tokumura A, Fukuzawa K, Yamada S, Tsukatani H. Stimulatory effect of lysophosphatidic acids on uterine smooth muscles of non-pregant rats. Arch Int Pharmacodyn Ther 1980; 245:74-83.

3 Simon MF, Chap H, Douste-Blazy L. Human platelet aggregation induced by 1-alkyl-lysophosphatidic acid and its analogs: a new group of phospholipid mediators? Biochem Biophys Res Commun 1982; 108: 1743-50.

4 Tsukatani H, Yamada S, Tokumura A, Miyamoto T, Takauchi K. Isolation of an acute hypotensive substance from bovine brain lipid fraction. Chem Pharm Bull (Tokyo) 1976; 24: 2294-300.

5 Moolenaar WH. Lysophosphatidic acid, a multifunctional phospholipid messenger. J Biol Chem 1995; 270: 12949-52.

6 van Corven EJ, Groenink A, Jalink K, Eichholtz T, Moolenaar WH. Lysophosphatidate-induced cell proliferation: identification and dissection of signaling pathways mediated by G proteins. Cell 1989; 59: 45-54.

7 Tigyi G, Miledi R. Lysophosphatidates bound to serum albumin activate membrane currents in Xenopus oocytes and neurite retraction in PC12 pheochromocytoma cells. J Biol Chem 1992; 267: 21360-7.

8 Hecht JH, Weiner JA, Post SR, Chun J. Ventricular zone gene-1 (vzg1) encodes a lysophosphatidic acid receptor expressed in neurogenic regions of the developing cerebral cortex. J Cell Biol 1996; 135: 1071-83.

9 An S, Bleu T, Hallmark OG, Goetzl EJ. Characterization of a novel subtype of human G protein-coupled receptor for lysophosphatidic acid. J Biol Chem 1998; 273: 7906-10.

10 Bandoh K, Aoki J, Hosono H, Kobayashi S, Kobayashi T, MurakamiMurofushi K, et al. Molecular cloning and characterization of a novel human G-protein-coupled receptor, EDG7, for lysophosphatidic acid. J Biol Chem 1999; 274: 27776-85.

11 Im DS, Heise CE, Harding MA, George SR, O'Dowd BF, Theodorescu D, et al. Molecular cloning and characterization of a lysophosphatidic acid receptor, Edg-7, expressed in prostate. Mol Pharmacol 2000; 57: 753-9.

12 Lynch KR, Im DS. Life on the edg. Trends Pharmacol Sci 1999; 20: 473-5.

13 Noguchi K, Ishii S, Shimizu T. Identification of p2y9/GPR23 as a novel $G$ protein-coupled receptor for Lysophosphatidic acid, structurally distant from the Edg family. J Biol Chem 2003; 278 : 25600-6.

14 Lee CW, Rivera R, Dubin AE, Chun J. $\mathrm{LPA}_{4} / \mathrm{GPR}^{23}$ is an LPA receptor utilizing $\mathrm{G}_{\mathrm{s}}, \mathrm{G}_{\mathrm{q}} / \mathrm{G}_{\mathrm{i}}$-mediated calcium signaling and $\mathrm{G}_{12 / 13}$-mediated Rho activation. J Biol Chem 2007; 282: 4310-7.

15 Lee CW, Rivera R, Gardell S, Dubin AE, Chun J. GPR92 as a New $\mathrm{G}_{12 / 13^{-}}$and $\mathrm{G}_{\mathrm{q}}$-coupled Lysophosphatidic Acid Receptor That Increases CAMP, LPA. J Biol Chem 2006; 281: 23589-97.

16 Kotarsky K, Boketoft A, Bristulf J, Nilsson NE, Norberg A, Hansson $\mathrm{S}$, et al. Lysophosphatidic Acid Binds to and Activates Gpr92, a G Protein-Coupled Receptor Highly Expressed in Gastro-Intestinal Lymphocytes. J Pharmacol Exp Ther 2006; 318: 619-628.

17 Yanagida K, Ishii S, Hamano F, Noguchi K, Shimizu T. LPA 4 /p2y9/ GPR23 mediates Rho-dependent morphological changes in a rat neuronal cell line. J Biol Chem 2007; 282: 5814-24.

18 Pasternack SM, von Kugelgen I, Aboud KA, Lee YA, Ruschendorf F, Voss $\mathrm{K}$, et al. G protein-coupled receptor P2Y5 and its ligand LPA are involved in maintenance of human hair growth. Nat Genet 2008; 40: 329-34.

19 Yanagida K, Masago K, Nakanishi H, Kihara Y, Hamano F, Tajima Y, et al. Identification and characterization of a novel lysophosphatidic acid receptor, p2y5/LPA . J Biol Chem 2009; 284: 17731-41.

20 Tabata K, Baba K, Shiraishi A, Ito M, Fujita N. The orphan GPCR GPR87 was deorphanized and shown to be a lysophosphatidic acid receptor. Biochem Biophys Res Commun 2007; 363: 861-6.

21 Murakami M, Shiraishi A, Tabata K, Fujita N. Identification of the orphan GPCR, P2Y(10) receptor as the sphingosine-1-phosphate and lysophosphatidic acid receptor. Biochem Biophys Res Commun 2008; 371: 707-12.

22 Noguchi K, Herr D, Mutoh T, Chun J. Lysophosphatidic acid (LPA) and its receptors. Curr Opin Pharmacol 2009; 9: 15-23.

23 Kitamura H, Okutani M, Arima N, Inoue A, Makide K, Aoki J. Identification of a novel receptor for lysophosphatidylserine. FASEB Summer Research Conference 2009: Poster \#24.

24 Olivera A, Spiegel S. Sphingosine-1-phosphate as second messenger in cell proliferation induced by PDGF and FCS mitogens. Nature 1993; 365: 557-60.

25 Choi $\mathrm{OH}$, Kim JH, Kinet JP. Calcium mobilization via sphingosine kinase in signalling by the Fc epsilon RI antigen receptor. Nature 1996; 380: 634-6.

26 Goodemote KA, Mattie ME, Berger A, Spiegel S. Involvement of a pertussis toxin-sensitive $G$ protein in the mitogenic signaling pathways of sphingosine 1-phosphate. J Biol Chem 1995; 270: 10272-7.

27 van Koppen C, Meyer zu Heringdorf M, Laser KT, Zhang C, Jakobs $\mathrm{KH}$, Bunemann $\mathrm{M}$, et al. Activation of a high affinity Gi proteincoupled plasma membrane receptor by sphingosine-1-phosphate. J Biol Chem 1996; 271: 2082-7. 
28 Bunemann M, Brandts B, zu Heringdorf DM, van Koppen CJ, Jakobs $\mathrm{KH}$, Pott $\mathrm{L}$. Activation of muscarinic $\mathrm{K}^{+}$current in guinea-pig atrial myocytes by sphingosine-1-phosphate. J Physiol 1995; 489: 701-7.

29 Okajima F, Tomura H, Sho K, Kimura T, Sato K, Im DS, et al. Sphingosine 1-phosphate stimulates hydrogen peroxide generation through activation of phospholipase $\mathrm{C}-\mathrm{Ca}^{2+}$ system in FRTL-5 thyroid cells: possible involvement of guanosine triphosphate-binding proteins in the lipid signaling. Endocrinology 1997; 138: 220-9.

30 Im DS, Fujioka T, Katada T, Kondo Y, Ui M, Okajima F. Characterization of sphingosine 1-phosphate-induced actions and its signaling pathways in rat hepatocytes. Am J Physiol 1997; 272: G1091-9.

31 Lee MJ, Van Brocklyn JR, Thangada S, Liu CH, Hand AR, Menzeleev R, et al. Sphingosine-1-phosphate as a ligand for the G protein-coupled receptor EDG-1. Science 1998; 279: 1552-5.

32 An S, Bleu T, Huang W, Hallmark OG, Coughlin SR, Goetzl EJ. Identification of cDNAs encoding two G protein-coupled receptors for lysosphingolipids. FEBS Lett 1997; 417: 279-82.

33 Okamoto H, Takuwa N, Yatomi Y, Gonda K, Shigematsu H, Takuwa Y. EDG3 is a functional receptor specific for sphingosine 1-phosphate and sphingosylphosphorylcholine with signaling characteristics distinct from EDG1 and AGR16. Biochem Biophys Res Commun 1999; 260: 203-8.

34 Van Brocklyn JR, Graler MH, Bernhardt G, Hobson JP, Lipp M, Spiegel S. Sphingosine-1-phosphate is a ligand for the $G$ protein-coupled receptor EDG-6. Blood 2000; 95: 2624-9.

35 Yamazaki Y, Kon J, Sato K, Tomura H, Sato M, Yoneya T, et al. Edg6 as a putative sphingosine 1-phosphate receptor coupling to $\mathrm{Ca}^{2+}$ signaling pathway. Biochem Biophys Res Commun 2000; 268: 583-9.

36 Im DS, Heise CE, Ancellin N, O'Dowd BF, Shei GJ, Heavens RP, et al. Characterization of a novel sphingosine 1-phosphate receptor, Edg-8. J Biol Chem 2000; 275: 14281-6.

37 Lynch KR, Macdonald TL. Structure-activity relationships of lysophosphatidic acid analogs. Biochim Biophys Acta 2002; 1582: 289-94.

38 Jalink K, Hengeveld T, Mulder S, Postma FR, Simon MF, Chap H, et al. Lysophosphatidic acid-induced $\mathrm{Ca}^{2+}$ mobilization in human A431 cells: structure-activity analysis. Biochem J 1995; 307: 609-16.

39 Gueguen G, Gaige B, Grevy JM, Rogalle P, Bellan J, Wilson M, et al. Structure-activity analysis of the effects of lysophosphatidic acid on platelet aggregation. Biochemistry 1999; 38: 8440-50.

40 Sugiura T, Tokumura A, Gregory L, Nouchi T, Weintraub ST, Hanahan DJ. Biochemical characterization of the interaction of lipid phosphoric acids with human platelets: comparison with platelet activating factor. Arch Biochem Biophys 1994; 311: 358-68.

41 Liliom K, Bittman R, Swords B, Tigyi G. N-palmitoyl-serine and $\mathrm{N}$-palmitoyl-tyrosine phosphoric acids are selective competitive antagonists of the lysophosphatidic acid receptors. Mol Pharmacol 1996; 50: 616-23.

42 Hooks SB, Ragan SP, Hopper DW, Honemann CW, Durieux ME, Macdonald TL, et al. Characterization of a receptor subtype-selective lysophosphatidic acid mimetic. Mol Pharmacol 1998; 53: 188-94.

43 Durgam GG, Virag T, Walker MD, Tsukahara R, Yasuda S, Liliom K, et al. Synthesis, structure-activity relationships, and biological evaluation of fatty alcohol phosphates as lysophosphatidic acid receptor ligands, activators of PPARgamma, and inhibitors of autotaxin. J Med Chem 2005; 48: 4919-30.

44 Lynch KR, Hopper DW, Carlisle SJ, Catalano JG, Zhang M, MacDonald TL. Structure/activity relationships in lysophosphatidic acid: the 2-hydroxyl moiety. Mol Pharmacol 1997; 52: 75-81.

45 Proll MA, Clark RB. Potent Gi-mediated inhibition of adenylyl cyclase by a phosphonate analog of monooleylphosphatidate. Mol Pharma- col 1991; 39: 740-4.

46 van der Bend RL, Brunner J, Jalink K, van Corven EJ, Moolenaar WH, van Blitterswijk WJ. Identification of a putative membrane receptor for the bioactive phospholipid, lysophosphatidic acid. EMBO J 1992; 11: 2495-501.

47 Xu Y, Casey G, Mills GB. Effect of lysophospholipids on signaling in the human Jurkat T cell line. J Cell Physiol 1995; 163: 441-50.

48 Bandoh K, Aoki J, Taira A, Tsujimoto M, Arai H, Inoue K. Lysophosphatidic acid (LPA) receptors of the EDG family are differentially activated by LPA species. Structure-activity relationship of cloned LPA receptors. FEBS Lett 2000; 478: 159-65.

49 Hasegawa Y, Erickson JR, Goddard GJ, Yu S, Liu S, Cheng KW, et al. Identification of a phosphothionate analogue of lysophosphatidic acid (LPA) as a selective agonist of the $\mathrm{LPA}_{3}$ receptor. J Biol Chem 2003; 278: 11962-9.

$50 \mathrm{Xu}$ Y, Qian L, Prestwich GD. Synthesis of monofluorinated analogues of lysophosphatidic acid. J Org Chem 2003; 68: 5320-30.

51 Xu Y, Aoki J, Shimizu K, Umezu-Goto M, Hama K, Takanezawa Y, et al. Structure-activity relationships of fluorinated lysophosphatidic acid analogues. J Med Chem 2005; 48: 3319-27.

52 Jiang G, Xu Y, Fujiwara Y, Tsukahara T, Tsukahara R, Gajewiak J, et al. Alpha-substituted phosphonate analogues of lysophosphatidic acid (LPA) selectively inhibit production and action of LPA. Chem Med Chem 2007; 2: 679-90.

53 Santos WL, Heasley BH, Jarosz R, Carter KM, Lynch KR, Macdonald TL. Synthesis and biological evaluation of phosphonic and thiophosphoric acid derivatives of lysophosphatidic acid. Bioorg Med Chem Lett 2004; 14: 3473-6.

54 Durgam GG, Tsukahara R, Makarova N, Walker MD, Fujiwara Y, Pigg $\mathrm{KR}$, et al. Synthesis and pharmacological evaluation of secondgeneration phosphatidic acid derivatives as lysophosphatidic acid receptor ligands. Bioorg Med Chem Lett 2006; 16: 633-40.

55 Williams JR, Khandoga AL, Goyal P, Fells Jl, Perygin DH, Siess W, et al. Unique Ligand Selectivity of the GPR92/LPA 5 Lysophosphatidate Receptor Indicates Role in Human Platelet Activation. J Biol Chem 2009; 284: 17304-19.

56 Virag T, Elrod DB, Liliom K, Sardar VM, Parrill AL, Yokoyama K, et al. Fatty alcohol phosphates are subtype-selective agonists and antagonists of lysophosphatidic acid receptors. Mol Pharmacol 2003; 63: 1032-42.

57 Gajewiak J, Tsukahara R, Tsukahara T, Fujiwara Y, Yu S, Lu Y, et al. Alkoxymethylenephosphonate analogues of (Lyso) phosphatidic acid stimulate signaling networks coupled to the $\mathrm{LPA}_{2}$ receptor. Chem Med Chem 2007; 2: 1789-98.

58 Tamaruya Y, Suzuki M, Kamura G, Kanai M, Hama K, Shimizu K, et al. Identifying specific conformations by using a carbohydrate scaffold: discovery of subtype-selective LPA-receptor agonists and an antagonist. Angew Chem Int Ed Engl 2004; 43: 2834-7.

59 Gududuru V, Zeng K, Tsukahara R, Makarova N, Fujiwara Y, Pigg KR, et al. Identification of Darmstoff analogs as selective agonists and antagonists of lysophosphatidic acid receptors. Bioorg Med Chem Lett 2006; 16: 451-6.

$60 \mathrm{Xu}$ Y, Tanaka M, Arai H, Aoki J, Prestwich GD. Alkyl lysophosphatidic acid and fluoromethylene phosphonate analogs as metabolicallystabilized agonists for LPA receptors. Bioorg Med Chem Lett 2004; 14: 5323-8.

61 Hooks SB, Santos WL, Im DS, Heise CE, Macdonald TL, Lynch KR. Lysophosphatidic acid-induced mitogenesis is regulated by lipid phosphate phosphatases and is Edg-receptor independent. J Biol Chem 2001; 276: 4611-21.

62 Oh DY, Yoon JM, Moon MJ, Hwang Jl, Choe H, Lee JY, et al. Identifica- 
tion of farnesyl pyrophosphate and $\mathrm{N}$-arachidonylglycine as endogenous ligands for GPR92. J Biol Chem 2008; 283: 21054-64.

63 Baker DL, Fujiwara Y, Pigg KR, Tsukahara R, Kobayashi S, Murofushi $\mathrm{H}$, et al. Carba analogs of cyclic phosphatidic acid are selective inhibitors of autotaxin and cancer cell invasion and metastasis. J Biol Chem 2006; 281: 22786-93.

64 Qian L, Xu Y, Simper T, Jiang G, Aoki J, Umezu-Goto M, et al. Phosphorothioate analogues of alkyl lysophosphatidic acid as $\mathrm{LPA}_{3}$ receptor-selective agonists. ChemMedChem 2006; 1: 376-83.

65 Endo T, Kano K, Motoki R, Hama K, Okudaira S, Ishida M, et al. Lysophosphatidylmethanol is a pan lysophosphatidic acid receptor agonist and is produced by autotaxin in blood. J Biochem 2009; 146: 283-93.

66 Heise CE, Santos WL, Schreihofer AM, Heasley BH, Mukhin YV, Macdonald TL, et al. Activity of 2-substituted lysophosphatidic acid (LPA) analogs at LPA receptors: discovery of a $\mathrm{LPA}_{1} / \mathrm{LPA}_{3}$ receptor antagonist. Mol Pharmacol 2001; 60: 1173-80.

67 Heasley BH, Jarosz R, Lynch KR, Macdonald TL. Initial structureactivity relationships of lysophosphatidic acid receptor antagonists: discovery of a high-affinity $\mathrm{LPA}_{1} / \mathrm{LPA}_{3}$ receptor antagonist. Bioorg Med Chem Lett 2004; 14: 2735-40.

68 Heasley BH, Jarosz R, Carter KM, Van SJ, Lynch KR, Macdonald TL. A novel series of 2-pyridyl-containing compounds as lysophosphatidic acid receptor antagonists: development of a nonhydrolyzable $\mathrm{LPA}_{3}$ receptor-selective antagonist. Bioorg Med Chem Lett 2004; 14 : 4069-74.

69 Fischer DJ, Nusser N, Virag T, Yokoyama K, Wang D, Baker DL, et al. Short-chain phosphatidates are subtype-selective antagonists of lysophosphatidic acid receptors. Mol Pharmacol 2001; 60: 776-84.

70 Ohta H, Sato K, Murata N, Damirin A, Malchinkhuu E, Kon J, et al. Ki16425, a subtype-selective antagonist for EDG-family lysophosphatidic acid receptors. Mol Pharmacol 2003; 64: 994-1005.

71 Liliom K, Tsukahara T, Tsukahara R, Zelman-Femiak M, Swiezewska E, Tigyi G. Farnesyl phosphates are endogenous ligands of lysophosphatidic acid receptors: inhibition of LPA GPCR and activation of PPARs. Biochim Biophys Acta 2006; 1761: 1506-14.

72 Fells Jl, Tsukahara R, Fujiwara Y, Liu J, Perygin DH, Osborne DA, et al. Identification of non-lipid $\mathrm{LPA}_{3}$ antagonists by virtual screening. Bioorg Med Chem 2008; 16: 6207-17.

73 Prestwich GD, Gajewiak J, Zhang H, Xu X, Yang G, Serban M. Phosphatase-resistant analogues of lysophosphatidic acid: agonists promote healing, antagonists and autotaxin inhibitors treat cancer. Biochim Biophys Acta 2008; 1781: 588-94.

74 Im DS, Clemens J, Macdonald TL, Lynch KR. Characterization of the human and mouse sphingosine 1-phosphate receptor, S1 $\mathrm{P}_{5}$ (Edg-8): structure-activity relationship of sphingosine1-phosphate receptors. Biochemistry 2001; 40: 14053-60.

75 Ancellin N, Hla T. Differential pharmacological properties and signal transduction of the sphingosine 1-phosphate receptors EDG-1, EDG3, and EDG-5. J Biol Chem 1999; 274: 18997-9002.

76 Candelore MR, Wright MJ, Tota LM, Milligan J, Shei GJ, Bergstrom JD, et al. Phytosphingosine 1-phosphate: a high affinity ligand for the $\mathrm{S}_{1} \mathrm{P}_{4} /$ Edg-6 receptor. Biochem Biophys Res Commun 2002; 297 : 600-6.

77 Lim HS, Park JJ, Ko K, Lee MH, Chung SK. Syntheses of sphingosine1-phosphate analogues and their interaction with EDG/S1P receptors. Bioorg Med Chem Lett 2004; 14: 2499-503.

78 Mandala S, Hajdu R, Bergstrom J, Quackenbush E, Xie J, Milligan J, et al. Alteration of lymphocyte trafficking by sphingosine-1-phosphate receptor agonists. Science 2002; 296: 346-9.

79 Brinkmann V, Davis MD, Heise CE, Albert R, Cottens S, Hof R, et al.
The immune modulator FTY720 targets sphingosine 1-phosphate receptors. J Biol Chem 2002; 277: 21453-7.

80 Im DS. Linking Chinese medicine and G-protein-coupled receptors. Trends Pharmacol Sci 2003; 24: 2-4.

81 Sanna MG, Liao J, Jo E, Alfonso C, Ahn MY, Peterson MS, et al. Sphingosine 1-phosphate (S1P) receptor subtypes $\mathrm{S}_{1} \mathrm{P}_{1}$ and $\mathrm{S}_{1} \mathrm{P}_{3}$, respectively, regulate lymphocyte recirculation and heart rate. J Biol Chem 2004; 279: 13839-48.

82 Jo E, Sanna MG, Gonzalez-Cabrera PJ, Thangada S, Tigyi G, Osborne $\mathrm{DA}$, et al. $\mathrm{S}_{1} \mathrm{P}_{1}$-selective in vivo - active agonists from high-throughput screening: off-the-shelf chemical probes of receptor interactions, signaling, and fate. Chem Biol 2005; 12: 703-15.

83 Li Z, Chen W, Hale JJ, Lynch CL, Mills SG, Hajdu R, et al. Discovery of potent 3,5-diphenyl-1,2,4-oxadiazole sphingosine-1-phosphate-1 $\left(\mathrm{S}_{1} \mathrm{P}_{1}\right)$ receptor agonists with exceptional selectivity against $\mathrm{S}_{1} \mathrm{P}_{2}$ and $\mathrm{S1P}_{3}$. J Med Chem 2005; 48: 6169-73.

84 Schurer SC, Brown SJ, Gonzalez-Cabrera PJ, Schaeffer MT, Chapman J, Jo E, et al. Ligand-binding pocket shape differences between sphingosine 1-phosphate (S1P) receptors $\mathrm{S}_{1} \mathrm{P}_{1}$ and $\mathrm{S}_{1} \mathrm{P}_{3}$ determine efficiency of chemical probe identification by ultrahigh-throughput screening. ACS Chem Biol 2008; 3: 486-98.

85 Gonzalez-Cabrera PJ, Jo E, Sanna MG, Brown S, Leaf N, Marsolais D, et al. Full pharmacological efficacy of a novel $\mathrm{S}_{1} \mathrm{P}_{1}$ agonist that does not require S1P-like headgroup interactions. Mol Pharmacol 2008; 74: 1308-18.

86 Pan S, Mi Y, Pally C, Beerli C, Chen A, Guerini D, et al. A monoselective sphingosine-1-phosphate receptor-1 agonist prevents allograft rejection in a stringent rat heart transplantation model. Chem Biol 2006; 13: 1227-34.

87 Zhu R, Snyder AH, Kharel Y, Schaffter L, Sun Q, Kennedy PC, et al. Asymmetric synthesis of conformationally constrained fingolimod analogues - discovery of an orally active sphingosine 1-phosphate receptor type-1 agonist and receptor type-3 antagonist. J Med Chem 2007; 50: 6428-35.

88 Fujishiro J, Kudou S, Iwai S, Takahashi M, Hakamata Y, Kinoshita M, et al. Use of sphingosine-1-phosphate 1 receptor agonist, KRP-203, in combination with a subtherapeutic dose of cyclosporine $A$ for rat renal transplantation. Transplantation 2006; 82: 804-12.

89 Song J, Matsuda C, Kai Y, Nishida T, Nakajima K, Mizushima T, et al. A novel sphingosine 1-phosphate receptor agonist, 2-amino-2propanediol hydrochloride (KRP-203), regulates chronic colitis in interleukin-10 gene-deficient mice. J Pharmacol Exp Ther 2008; 324: $276-83$

90 Hanessian S, Charron G, Billich A, Guerini D. Constrained azacyclic analogues of the immunomodulatory agent FTY720 as molecular probes for sphingosine 1-phosphate receptors. Bioorg Med Chem Lett 2007; 17: 491-4.

91 Himmel HM, Meyer Zu Heringdorf D, Graf E, Dobrev D, Kortner A, Schuler S, et al. Evidence for Edg-3 receptor-mediated activation of I(K.ACh) by sphingosine-1-phosphate in human atrial cardiomyocytes. Mol Pharmacol 2000; 58: 449-54.

92 Niedernberg A, Scherer CR, Busch AE, Kostenis E. Comparative analysis of human and rat $\mathrm{S}_{1} \mathrm{P}_{5}$ (edg8): differential expression profiles and sensitivities to antagonists. Biochem Pharmacol 2002; 64: 1243-50.

93 Ikeda H, Satoh H, Yanase M, Inoue Y, Tomiya T, Arai M, et al. Antiproliferative property of sphingosine 1-phosphate in rat hepatocytes involves activation of Rho via Edg-5. Gastroenterology 2003; 124: 459-69.

94 Ohmori T, Yatomi Y, Osada M, Kazama F, Takafuta T, Ikeda H, et al. Sphingosine 1-phosphate induces contraction of coronary artery 
smooth muscle cells via S1P . Cardiovasc Res 2003; 58: 170-7.

95 Davis MD, Clemens JJ, Macdonald TL, Lynch KR. Sphingosine 1-phosphate analogs as receptor antagonists. J Biol Chem 2005; 280: 9833-41.

96 Sanna MG, Wang SK, Gonzalez-Cabrera PJ, Don A, Marsolais D, Matheu MP, et al. Enhancement of capillary leakage and restoration of lymphocyte egress by a chiral $\mathrm{S}_{1} \mathrm{P}_{1}$ antagonist in vivo. Nat Chem Biol 2006; 2: 434-41.

97 Foss FW Jr, Snyder AH, Davis MD, Rouse M, Okusa MD, Lynch KR, et al. Synthesis and biological evaluation of gamma-aminophosphonates as potent, subtype-selective sphingosine 1-phosphate receptor agonists and antagonists. Bioorg Med Chem 2007; 15: 663-77.

98 Waters CM, Long J, Gorshkova I, Fujiwara Y, Connell M, Belmonte $\mathrm{KE}$, et al. Cell migration activated by platelet-derived growth factor receptor is blocked by an inverse agonist of the sphingosine 1-phosphate receptor-1. FASEB J 2006; 20: 509-11.

99 Yonesu K, Ohnuki T, Ono Y, Takatsu T, Nara F. Ascotricins A and $B$, novel antagonists of sphingosine-1-phosphate receptor 1 from Ascotricha chartarum Berk. SANK 14186. J Antibiot (Tokyo) 2009; 62: 359-64.

100 Yonesu K, Kawase Y, Inoue T, Takagi N, Tsuchida J, Takuwa Y, et al. Involvement of sphingosine-1-phosphate and $\mathrm{S}_{1} \mathrm{P}_{1}$ in angiogenesis: analyses using a new $\mathrm{S}_{1} \mathrm{P}_{1}$ antagonist of non-sphingosine-1-phosphate analog. Biochem Pharmacol 2009; 77: 1011-20.

101 Goetzl EJ, Dembrow D, Van Brocklyn JR, Graler M, Huang MC. An IgMkappa rat monoclonal antibody specific for the type 1 sphingosine 1-phosphate $\mathrm{G}$ protein-coupled receptor with antagonist and agonist activities. Immunol Lett 2004; 93: 63-9.

102 Koide Y, Hasegawa T, Takahashi A, Endo A, Mochizuki N, Nakagawa M, et al. Development of novel EDG3 antagonists using a 3D database search and their structure-activity relationships. J Med Chem 2002; 45: 4629-38.

103 Jongsma M, Hendriks-Balk MC, Michel MC, Peters SL, Alewijnse AE. BML-241 fails to display selective antagonism at the sphingosine-1phosphate receptor, $\mathrm{S}_{1} \mathrm{P}_{3}$. Br J Pharmacol 2006; 149: 277-82.

104 Koide Y, Uemoto K, Hasegawa T, Sada T, Murakami A, Takasugi H, et al. Pharmacophore-based design of sphingosine 1-phosphate-3 receptor antagonists that include a 3,4-dialkoxybenzophenone scaffold. J Med Chem 2007; 50: 442-54.

105 Zhang H, Desai NN, Olivera A, Seki T, Brooker G, Spiegel S. Sphingosine-1-phosphate, a novel lipid, involved in cellular proliferation. J Cell Biol 1991; 114: 155-67.

106 Rosen H, Gonzalez-Cabrera PJ, Sanna MG, Brown S. Sphingosine 1-phosphate receptor signaling. Annu Rev Biochem 2009; 78: 74368.

107 Rivera R, Chun J. Biological effects of lysophospholipids. Rev Physiol Biochem Pharmacol 2008; 160: 25-46.

108 Skoura A, Hla T. Lysophospholipid receptors in vertebrate development, physiology, and pathology. J Lipid Res 2009; 50: S293-8.

109 Ishii I, Fukushima N, Ye X, Chun J. Lysophospholipid receptors: signaling and biology. Annu Rev Biochem 2004; 73: 321-54.

110 Brinkmann V, Lynch KR. FTY720: targeting G-protein-coupled receptors for sphingosine 1-phosphate in transplantation and autoimmunity. Curr Opin Immunol 2002; 14: 569-75.

111 Brown BA, Kantesaria PP, McDevitt LM. Fingolimod: a novel immunosuppressant for multiple sclerosis. Ann Pharmacother 2007; 41: 1660-8.

112 Swaney JS, Chapman C, Correa LD, Bain G, Bundey RA, Prodanovich $\mathrm{PP}$, et al. A novel, small molecule, $\mathrm{LPA}_{1}$ receptor-selective antagonist, inhibits bleomycin-induced inflammation and fibrosis in the mouse. FASEB Summer Research Conference. 2009.

113 Nagiec M, Funckes-Shippy C, Hiebsch R, Radi Z, Walker J, Gierse $J$, et al. Targeting S1P signaling for inflammation. FASEB Summer Research Conference. 2009.

114 Sabbadini R, Cavalli A, Wocjiak J, Visentin B, Matteo R, Campbell M, et al. Antibodies against bioactive lipids. FASEB Summer Research Conference. 2009.

115 Caballero S, Swaney J, Moreno K, Afzal A, Kielczewski J, Stoller G, et al. Anti-sphingosine-1-phosphate monoclonal antibodies inhibit angiogenesis and sub-retinal fibrosis in a murine model of laserinduced choroidal neovascularization. Exp Eye Res 2009; 88: $367-$ 77.

116 Prestwich GD. Dual activity LPA receptor pan-antagonist/antotaxin inhibitors as anti-cancer agents: evaluation in vivo using engineered human tumors. FASEB Summer Research Conference. 2009.

117 Zhang H, Xu X, Gajewiak J, Tsukahara R, Fujiwara Y, Liu J, et al. Dual activity lysophosphatidic acid receptor pan-antagonist/autotaxin inhibitor reduces breast cancer cell migration in vitro and causes tumor regression in vivo. Cancer Res 2009; 69: 5441-9.

118 Goetzl EJ, Lynch KR. Preface: the omnific lysophospholipid growth factors. Ann N Y Acad Sci 2000; 905: xi-xiv.

119 Chun J, Goetzl EJ, Hla T, Igarashi Y, Lynch KR, Moolenaar W, et al. International Union of Pharmacology. XXXIV. Lysophospholipid Receptor Nomenclature. Pharmacol Rev 2002; 54: 265-9.

120 Fells Jl, Tsukahara R, Liu J, Tigyi G, Parrill AL. Structure-based drug design identifies novel $\mathrm{LPA}_{3}$ antagonists. Bioorg Med Chem 2009; 17: 7457-64.

121 Bolli MH, Abele S, Binkert C, Bravo R, Buchmann S, Bur D, et al. 2-imino-thiazolidin-4-one derivatives as potent, orally active $\mathrm{S}_{1} \mathrm{P}_{1}$ receptor agonists. J Med Chem 2010; 53: 4198-211.

122 An S, Bleu T, Zheng Y, Goetzl EJ. Recombinant human G proteincoupled lysophosphatidic acid receptors mediate intracellular calcium mobilization. Mol Pharmacol 1998; 54: 881-8.

123 Murakami A, Takasugi H, Ohnuma S, Koide Y, Sakurai A, Takeda S, et al. Sphingosine 1-phosphate $(\mathrm{S} 1 \mathrm{P})$ regulates vascular contraction via $\mathrm{S}_{1} \mathrm{P}_{3}$ receptor: investigation based on a new $\mathrm{S}_{1} \mathrm{P}_{3}$ receptor antagonist. Mol Pharmacol 2010; 77: 704-13.

124 Kim K, Kim YL, Sacket SJ, Kim HL, Han M, Park DS, et al. Sphingosine 1-phosphate (S1P) induces shape change in rat C6 glioma cells through the $\mathrm{S}_{1} \mathrm{P}_{2}$ receptor: development of an agonist for $\mathrm{S} 1 \mathrm{P}$ receptors. J Pharm Pharmacol 2007; 59: 1035-41. 